\title{
A COMPARISON THEOREM FOR THE FIRST DIRICHLET EIGENVALUE OF A DOMAIN IN A KAEHLER SUBMANIFOLD
}

\author{
FRANCISCO J. CARRERAS, FERNANDO GIMÉNEZ and \\ VICENTE MIQUEL
}

(Received 1 March 1991; revised 28 October 1991)

Communicated by K. C. H. MacKenzie

\begin{abstract}
We give a sharp lower bound for the first eigenvalue of the Dirichlet eigenvalue problem on a domain of a complex submanifold of a Kaehler manifold with curvature bounded from above. The bound on the first eigenvalue is given as a function of the extrinsic outer radius and the bounds on the curvature, and it is attained only on geodesic spheres of a space of constant holomorphic sectional curvature embedded in the Kaehler manifold as a totally geodesic submanifold.
\end{abstract}

1991 Mathematics subject classification (Amer. Math. Soc.): primary 58 G 20; secondary 58 G 30, 53 C 21, 53 C 55.

\section{Introduction}

Given a compact connected $n$-dimensional Riemannian manifold $V$ with boundary $\partial V$, let $\lambda_{1}(V)$ be the first eigenvalue of the Dirichlet eigenvalue problem

$$
\Delta f=\lambda f \text { on } V \quad \text { and } \quad f=0 \text { on } \partial V .
$$

For $\mathrm{V}$ a domain in a Riemannian manifold, many interesting comparison theorems for $\lambda_{1}(V)$ have been proved in the last years. Many of them can be (C) 1994 Australian Mathematical Society 0263-6115/94 \$A2.00+0.00 Work partially supported by a DGICYT Grant No. PB91-0324. 
found in [2], where many references are given. In [5] a comparison theorem has been given for domains in Kaehler manifolds. In [3, Corollary 3], and [12, Corollary C], Cheng, $\mathrm{Li}$, and Yau, and Markvorsen, get a comparison theorem for $\lambda_{1}(V)$, where $V$ is a domain in a minimal submanifold of a manifold with sectional curvature bounded from above. We prove here a similar result by restricting the family of pairs (manifold, submanifold), and getting a greater lower bound for $\lambda_{1}(V)$.

Let $M$ be a Kaehler manifold of real dimension $2 n$, with Riemannian metric $\langle$,$\rangle , and almost complex structure J$, and let $p$ be a point of $M$. Let $\gamma(t)$ be a geodesic starting from $p$, parametrized with respect to its arc length. We shall consider, on the orthogonal complement $\left\{\gamma^{\prime}(t)\right\}^{\perp}$ of $\gamma^{\prime}(t)$ in $T_{\gamma(t)} M$, the operators $S(t)$, the Weingarten map of a geodesic sphere of centre $p$ and radius $r$, and $R(t)$, the curvature operator along $\gamma(t)$, as in [6, page 37]. Let us denote by $S_{\lambda}(t)$ and $R_{\lambda}(t)$ the corresponding operators on the complex space form $\mathbb{K}^{n}(\lambda)$ of constant holomorphic sectional curvature $4 \lambda\left(\mathbb{K}^{n}(\lambda)=\mathbb{C} P^{n}(\lambda)\right.$ if $\lambda>0$ and $\mathbb{K}^{n}(\lambda)=\mathbb{C} H^{n}(\lambda)$ if $\lambda<0$ ). Let us denote also by $S(t), R(t), S_{\lambda}(t), R_{\lambda}(t)$ the symmetric bilinear forms associated to these operators and the metrics on $M$ or $\mathbb{K}^{n}(\lambda)$.

Given two quadratic forms, $A$ and $B$, we shall say that $A \geq B$ if $A-B$ is positive semidefinite.

Let $P$ be a complex submanifold of $M$ of real dimension $2 q, \Omega$ a compact domain in $P, p \in \Omega$, and let $r_{p}: P \rightarrow \mathbb{R}$ be the extrinsic distance to $p$ $\left(r_{p}(x)=d(p, x)\right.$, where $d$ is the distance function in $\left.M\right)$. The extrinsic outer radius of $\Omega$ at $p$ is defined by

$$
a=\sup \left\{r_{p}(z), z \in \Omega\right\} .
$$

The extrinsic ball of centre $p$ and radius $a$ is defined by $B_{r}(p)=\{m \in P \mid$ $\left.r_{p}(m) \leq a\right\}$. Obviously, $\Omega \subset B_{a}(p) . B_{a}^{\lambda, q}$ will denote a geodesic ball of radius $a$ in $\mathbb{K}^{q}(\lambda)$.

In this paper we shall prove the following theorem:

THEOREM 1.1. Let $M, P, \Omega, p$ and $a$ be as above. Let us assume that on every geodesic $\gamma(t)$ of $M$ starting from $p, R(t) \leq R_{\lambda}(t)$. If $\operatorname{cut}(p) \cap B_{a}(p)=\emptyset$ (and $a<\pi /(2 \sqrt{\lambda})$ if $\lambda>0)$, then

$$
\lambda_{1}(\Omega) \geq \lambda_{1}\left(B_{a}^{\lambda, q}\right) .
$$

Moreover, if the equality holds $\Omega$ is holomorphically isometric to a geodesic ball of radius a in $\mathbb{K}^{q}(\lambda)$. If the hypothesis $R(t) \leq R_{\lambda}(t)$ holds for every $p \in \Omega$, then the equality in (1.1) implies also that $\Omega$ is totally geodesic in $M$. 
REMARK. This result is also valid if we take as $a$ the infimum of the extrinsic outer radii at $p, p \in \Omega$, and the hypothesis $R(t) \leq R_{\lambda}(t)$ holds for every $p \in \Omega$.

Similar results for intrinsic balls are easier to prove. However, since the extrinsic outer radius is always less than the intrinsic one, the bounds obtained are worse.

After stating the result an immediate question arises: does there exist a Kaehler manifold $M$ satisfying $R(t) \leq R_{\lambda}(t)$ and such that the supremum of the sectional curvature of $M$ is greater than $\lambda$ ? In Section 4 we shall give a few examples of such manifolds for $\lambda>0$.

\section{Preliminary results}

LEMMA 2.1. Let $M$ be a Kaehler manifold of real dimension $2 n, p \in M$, and $\gamma(t), R(t), S(t), R_{\lambda}(t), S_{\lambda}(t)$ as in Section 1. If $R(t) \leq R_{\lambda}(t)$, then

$$
S(t) \leq S_{\lambda}(t)
$$

for every $t \in\left(0, \operatorname{conj}_{\gamma}(m)\right)$, where $\operatorname{conj}_{\gamma}(m)$ is the first $t \in \mathbb{R}^{+}$such that $\gamma(t)$ is a conjugate point of $m$ along $\gamma$.

Moreover, the equality in (2.1.1) for $t \in(0, r), r<\operatorname{cut}(m)$, implies the existence of a holomorphic isometry between $B_{r}(m)$ and $B_{r}^{\lambda, n}$ which takes $S_{r}(m)$ onto $S_{r}^{\lambda, n}=\partial B_{r}^{\lambda, n}$.

PROOF. The inequality (2.1.1) is a consequence of [13, Theorem 5]. The characterization of the equality follows from [11, Theorem 8] or [4, Proposition 2.5].

LEMMA 2.2. ([9] or [7]) Hess $r_{p}(X, Y)=\nabla^{2} r_{p}(X, Y)=-\left\langle S\left(r_{p}\right) X, Y\right\rangle$, where $\nabla$ is the covariant derivative in $M$ and Hess is the Hessian acting on functions.

LEMMA 2.3. ([8]) Let $P$ and $M$ be as above, $g: M \rightarrow \mathbb{R}$ a smooth function, $f=\left.g\right|_{P}$ the restriction of $g$ to $P$, and $\alpha$ the second fundamental form of $P$ in $M$ (with the sign convention given in [10] and [6]). If $\nabla$ is the covariant derivative in $P$ and $\bar{\nabla}$ the covariant derivative in $M$, then

$$
\nabla^{2} f(X, Y)=\bar{\nabla}^{2} g(X, Y)+\langle\overline{\operatorname{grad}} g, \alpha(X, Y)\rangle,
$$

where $\overline{\text { grad }}$ means the gradient in $M$. 
From Lemmas 2.2 and 2.3 and the fact that $P$ is a complex submanifold, we get

$$
\Delta r_{p}=\bar{\Delta} r_{p}
$$

where $\Delta$ (respectively $\bar{\Delta}$ ) is the Laplacian in $P$ (respectively the trace in $T P$ of $\left.\bar{\nabla}^{2} r_{p}\right)$.

Proposition 2.5. Assume that $R(t) \leq R_{\lambda}(t)$ and that $s: \mathbb{R}^{+} \rightarrow \mathbb{R}^{+}$is a monotone increasing function. Then, denoting $r_{p}$ by $r$,

(2.5.1) $\Delta(s \circ r) \leq\left|\overline{\operatorname{grad}} r^{\top}\right|^{2}\left(-s^{\prime \prime}(r)-(2 \mu-v) s^{\prime}(r)\right)+2 q \mu(r) s^{\prime}(r)$,

where

$$
\begin{aligned}
& \mu(r)=\left\{\begin{array}{ll}
-\sqrt{\lambda} \cot (\sqrt{\lambda} r) & \text { if } \lambda>0 \\
-\sqrt{|\lambda|} \operatorname{coth}(\sqrt{|\lambda|} r) & \text { if } \lambda<0
\end{array},\right. \\
& \nu(r)=\left\{\begin{array}{ll}
-2 \sqrt{\lambda} \cot (2 \sqrt{\lambda} r) & \text { if } \lambda>0 \\
-2 \sqrt{|\lambda|} \operatorname{coth}(2 \sqrt{|\lambda|} r) & \text { if } \lambda<0
\end{array} .\right.
\end{aligned}
$$

and $\overline{\operatorname{grad}} r^{\top}$ is the component of the vector $\overline{\operatorname{grad}} r$ tangent to $P$.

PROOF. First let us recall ([6, page 138]) that

$$
S_{\lambda}(r) X=\mu\left(X-\left\langle X, J \partial_{r}\right\rangle J \partial_{r}\right)+\nu\left\langle X, J \partial_{r}\right\rangle J \partial_{r} .
$$

Given a point $p \in P$, let $\left\{E_{i}\right\}_{i=1}^{2 q}$ be a set of unit vectors such that $\bar{\nabla}_{E_{i}} E_{j}(p)=$ 0 and $\left\{E_{1}(p), \cdots, E_{2 q}(p)\right\}$ is a $J$-orthonormal basis of $T_{p} P$. Then, from (2.2), (2.3) and the fact that $P$ is a complex submanifold, we get

$$
\begin{aligned}
\Delta(s \circ r)(p) & =-\sum_{i=1}^{2 q}\left\langle\bar{\nabla}_{E_{i}} \operatorname{grad}(s \circ r), E_{i}\right\rangle(p)=-\sum_{i=1}^{2 q}\left\langle\bar{\nabla}_{E_{i}} s^{\prime} \partial_{r}, E_{i}\right\rangle(p) \\
& =-\sum_{i=1}^{2 q}\left\langle s^{\prime \prime}\left\langle\operatorname{grad} r, E_{i}\right\rangle \partial_{r}+s^{\prime} \bar{\nabla}_{E_{i}} \partial_{r}, E_{i}\right\rangle(p) \\
& =\left\{-s^{\prime \prime} \sum_{i=1}^{2 q}\left\langle\overline{\operatorname{grad}} r, E_{i}\right\rangle^{2}+s^{\prime} \sum_{i=1}^{2 q}\left\langle S(r) E_{i}, E_{i}\right\rangle\right\}(p) .
\end{aligned}
$$

where grad means the gradient in $P$. But from (2.1) and (2.5.2), 


$$
\begin{aligned}
\sum_{i=1}^{2 q}\left\langle S(r) E_{i},\right. & \left.E_{i}\right\rangle(p) \\
= & \left.\sum_{i=1}^{2 q}\left\{S(r)\left(E_{i}-\left\langle E_{i}, \partial_{r}\right\rangle \partial_{r}\right), E_{i}\right\rangle+\left\langle E_{i}, \partial_{r}\right\rangle\left\langle S(r) \partial_{r}, E_{i}\right\rangle\right\}(p) \\
= & \sum_{i=1}^{2 q}\left\langle S(r)\left\{\left(E_{i}-\left\langle E_{i}, \partial_{r}\right\rangle \partial_{r}-\left\langle E_{i}, J \partial_{r}\right\rangle J \partial_{r}\right)+\left\langle E_{i}, J \partial_{r}\right\rangle J \partial_{r}\right\}\right. \\
& \left.\left(E_{i}-\left\langle E_{i}, \partial_{r}\right\rangle \partial_{r}-\left\langle E_{i}, J \partial_{r}\right\rangle J \partial_{r}\right)+\left\langle E_{i}, J \partial_{r}\right\rangle J \partial_{r}\right\rangle(p)
\end{aligned}
$$

$$
\begin{aligned}
\leq & \sum_{i=1}^{2 q}\left(\mu(r)\left|E_{i}-\left\langle E_{i}, \partial_{r}\right\rangle \partial_{r}-\left\langle E_{i}, J \partial_{r}\right\rangle J \partial_{r}\right|^{2}(p)\right. \\
& \left.+v(r)\left|\left\langle E_{i}, J \partial_{r}\right\rangle\right|^{2}(p)\right) \\
= & \sum_{i=1}^{2 q}\left(1-\left\langle E_{i}, \partial_{r}\right\rangle^{2}-\left\langle J E_{i}, \partial_{r}\right\rangle^{2}\right)(p) \mu(r)+\sum_{i=1}^{2 q}\left\langle J E_{i}, \partial_{r}\right\rangle^{2}(p) v(r) \\
= & 2 q \mu(r)-2 \sum_{i=1}^{2 q}\left\langle\overline{\operatorname{grad}} r, E_{i}\right\rangle^{2} \mu(r)+\sum_{i=1}^{2 q}\left\langle\overline{\operatorname{grad}} r, E_{i}\right\rangle^{2} \nu(r) \\
= & 2 q \mu(r)+\left|\overline{\operatorname{grad}} r^{\top}\right|^{2}(-2 \mu(r)+v(r)) .
\end{aligned}
$$

And the result follows from (2.5.3) and (2.5.4) and the hypothesis $s^{\prime} \geq 0$.

Now, if the function $s$ satisfies

$$
s^{\prime \prime}+(2 \mu-v) s^{\prime}=0,
$$

then (2.5.1) simplifies to become

$$
\Delta(s \circ r) \leq 2 q \mu(r) s^{\prime}(r) .
$$

\section{Proof of Theorem 1.1}

Since $\Omega \subset B_{a}(p)$, we have ([2, page 18]) that $\lambda_{1}(\Omega) \geq \lambda_{1}\left(B_{a}(p)\right)$, and the equality implies that $\Omega=B_{a}(p)$. Then, it is enough to prove the theorem for $\Omega=B_{a}(p)$.

Let $\phi$ be the eigenfunction of (1.1) corresponding to $\lambda_{1}\left(B_{a}^{\lambda, q}\right)$. It is well known ([1]) that it is a radial function, and that we can take it positive. Then we 
can write $\phi(y)=\phi(d(x, y))$, and define $\phi$ on $\Omega$ by $\phi(m)=\phi\left(r_{p}(m)\right)$. Now, using the inverse function of $s$, we can consider $\phi$ as a function of $s$ as well as a function of $r_{p}$. By a computation similar to (2.5.3), and denoting by ${ }^{\circ}$ the derivative with respect to $s$ and by ' the derivative with respect to $r$, we get:

$$
\begin{aligned}
\Delta \phi\left(s \circ r_{p}(y)\right) & =-\phi^{\prime \prime} \sum_{i=1}^{2 q}\left\langle\overline{\operatorname{grad}} r, E_{i}\right\rangle^{2}+\phi^{\prime} \bar{\Delta} r \\
& =-\ddot{\phi} s^{2} \sum_{i=1}^{2 q}\left\langle\overline{\operatorname{grad}} r, E_{i}\right\rangle^{2}+\dot{\phi}\left(-s^{\prime \prime} \sum_{i=1}^{2 q}\left\langle\overline{\operatorname{grad}} r, E_{i}\right\rangle+s^{\prime} \bar{\Delta} r\right) \\
& =-\ddot{\phi} s^{2} \sum_{i=1}^{2 q}\left\langle\overline{\operatorname{grad}} r, E_{i}\right\rangle^{2}+\dot{\phi} \Delta s
\end{aligned}
$$

If

$$
\ddot{\phi} \geq 0 \quad \text { and } \quad \dot{\phi} \leq 0 \text {, }
$$

and (2.6) holds, then

$$
\begin{aligned}
\Delta \phi(s(r)) & \geq-\ddot{\phi} s^{2}+\dot{\phi} \Delta s \\
& \geq-\ddot{\phi} s^{2}+2 q \mu \dot{\phi} s^{\prime}=\Delta_{\lambda, q} \phi=\lambda_{1}\left(B_{a}^{\lambda, q}\right) \phi
\end{aligned}
$$

where we have used (2.7) in the second inequality.

A solution of $(2.6)$ is

$$
s^{\prime}=\left\{\begin{array}{ll}
\frac{1}{2} \tan (\sqrt{\lambda} r) & \text { if } \lambda>0 \\
\frac{1}{2} \tanh (\sqrt{|\lambda|} r) & \text { if } \lambda<0
\end{array} \quad s= \begin{cases}-\frac{1}{2 \sqrt{\lambda}} \log \cos (\sqrt{\lambda} r) & \text { if } \lambda>0 \\
-\frac{1}{2 \sqrt{\lambda}} \log \cosh (\sqrt{|\lambda|} r) & \text { if } \lambda<0 .\end{cases}\right.
$$

The second inequality in (3.1) follows, since

$$
\dot{\phi}=\phi^{\prime} \frac{1}{s^{\prime}} \leq 0,
$$

for $s^{\prime} \geq 0$ and $\phi^{\prime} \leq 0$ (cf. [1]). If we prove the first, we have (3.2), and then, by Barta's lemma $\left(\left[2\right.\right.$, page 70]), we get $\lambda_{1}\left(B_{a}(p)\right) \geq \inf _{\Omega}(\Delta \phi) / \phi \geq \lambda_{1}\left(B_{a}^{\lambda, q}\right)=\lambda_{1}^{c}$ if $\phi$ is strictly positive in the interior of $B_{a}(p)$, which is true by [1]. Then it is sufficient to prove that $\ddot{\phi} \geq 0$.

By differentiating (3.4), taking account of (2.6), we get

$$
\ddot{\phi}=\frac{1}{s^{2}}\left(\phi^{\prime \prime}-(\nu-2 \mu) \phi^{\prime}\right) \text {. }
$$


On the other hand, from (2.5.3) and the expression of $S(r)$ in $\mathbb{K}^{q}(\lambda)$ we get

$$
-\phi^{\prime \prime}+((2 q-2) \mu+v) \phi^{\prime}=\Delta_{\lambda, q} \phi=\lambda_{1}^{c} \phi .
$$

Then

$$
\ddot{\phi}=\frac{1}{s^{\prime 2}}\left(-\lambda_{1}^{c} \phi+2 q \mu \phi^{\prime}\right) .
$$

Since $\phi$ is radial, we must have (see also [1])

$$
\phi^{\prime}(0)=0 .
$$

Let us first consider the case $\lambda>0$. From (3.6) it follows that

$$
\begin{aligned}
\phi^{\prime \prime} & =-\lambda_{1}^{c} \phi(0)+\sqrt{\lambda} \lim _{r \rightarrow 0} \frac{1-2 q \cos ^{2}(\sqrt{\lambda} r)}{\cos (\sqrt{\lambda} r)} \cdot \lim _{r \rightarrow 0} \frac{\phi^{\prime}}{\sin (\sqrt{\lambda} r)} \\
& =-\lambda_{1}^{c} \phi(0)+(1-2 q) \phi^{\prime \prime}(0)
\end{aligned}
$$

whence

$$
\phi^{\prime \prime}(0)=-\frac{\lambda_{1}^{c}}{2 q} \phi(0)<0
$$

By differentiating (3.6) we get

$$
-\phi^{\prime \prime \prime}+\lambda \frac{2(q-1) \cos ^{2}(\sqrt{\lambda} r)+1}{\sin ^{2}(\sqrt{\lambda} r) \cos ^{2}(\sqrt{\lambda} r)} \phi^{\prime}-\sqrt{\lambda} \frac{2 q \cos (\sqrt{\lambda} r)-1}{\sin (\sqrt{\lambda} r) \cos (\sqrt{\lambda} r)} \phi^{\prime \prime}=\lambda_{1}^{c} \phi^{\prime}
$$

Let us define

$$
g(r)=\lambda_{1}^{c} \phi-2 q \mu \phi^{\prime}=\lambda_{1}^{c} \phi+2 q \sqrt{\lambda} \frac{\phi_{r}}{\tan (\sqrt{\lambda} r)} .
$$

By computing limits for $r \rightarrow 0$, using l'Hôpital rule and (3.9), we get

$$
\begin{aligned}
g(0) & =\lambda_{1}^{c} \phi(0)+2 q \sqrt{\lambda} \lim _{r \rightarrow 0} \frac{4 \phi^{\prime}}{\sqrt{\lambda}\left(1+\tan ^{2}(\sqrt{\lambda} r)\right)} \\
& =\lambda_{1}^{c} \phi(0)+2 q \phi^{\prime \prime}(0)=0 .
\end{aligned}
$$

By differentiating (3.11), and using (3.6),

$$
\frac{\partial g}{\partial r}=\lambda_{1}^{c} \phi_{r}-2 q \sqrt{\lambda} \frac{g(r)}{\tan (\sqrt{\lambda} r)}
$$


We now take limits when $r \rightarrow 0$, and use (3.8) to get

$$
\frac{\partial g}{\partial r}(0)=-2 q \frac{\partial g}{\partial r}(0) \text {. }
$$

Hence,

$$
\frac{\partial g}{\partial r}(0)=0
$$

The second derivative of $g$ is

$$
\begin{aligned}
\frac{\partial^{2} g}{\partial r^{2}}=\lambda_{1}^{c} \phi^{\prime \prime}+2 q \sqrt{\lambda} \cos (\sqrt{\lambda} r) \frac{\phi^{\prime \prime \prime}}{\sin (\sqrt{\lambda} r)}-4 q \lambda \frac{\phi^{\prime \prime}}{\sin ^{2}(\sqrt{\lambda} r)} & \\
& +4 q \lambda^{3 / 2} \cos (\sqrt{\lambda} r) \frac{\phi^{\prime}}{\sin ^{3}(\sqrt{\lambda} r)},
\end{aligned}
$$

and from this expression, (3.6) and (3.10), we get

$$
\frac{\partial^{2} g}{\partial r^{2}}=\lambda_{1}^{c} \phi^{\prime \prime}+2 q \sqrt{\lambda} \lambda_{1}^{c} \frac{\phi^{\prime}}{\tan (\sqrt{\lambda} r)}+2 q \lambda\left(1+2 q \cos ^{2}(\sqrt{\lambda} r)\right) \frac{g(r)}{\sin ^{2}(\sqrt{\lambda} r)} .
$$

Since $g(0)=0$ and $\partial g / \partial r(0)=0$ we get, by l'Hôpital rule,

$$
\frac{\partial^{2} g}{\partial r^{2}}(0)=\lambda_{1}^{c} \phi^{\prime \prime}(0)-2 q \sqrt{\lambda} \lambda_{1}^{c} \phi^{\prime \prime}(0)+q(1+2 q) \frac{\partial^{2} g}{\partial r^{2}}(0),
$$

whence

$$
\frac{\partial^{2} g}{\partial r^{2}}(0)=\frac{(2 q-1) \lambda_{1}^{c}}{q(1+2 q)-1} \phi^{\prime \prime}(0)<0,
$$

and from (3.5), using l'Hôpital rule again,

$$
\ddot{\phi}(0)=-\frac{2}{\lambda} \frac{(2 q-1) \lambda_{1}^{c}}{q(1+2 q)-1} \phi^{\prime \prime}(0)>0 .
$$

A similar computation gives $\ddot{\phi}(0)>0$ for $\lambda<0$.

Let $s_{0}$ be the first positive zero of $\ddot{\phi}$. Since $\ddot{\phi}(0)>0, \ddot{\phi}(s)>0$ for $s<s_{0}$, $\dot{\phi}$ has either a maximum or a inflexion point at $s_{0}$. On the other hand, by differentiating (3.2), we get

$$
\lambda_{1}^{c} \dot{\phi}=-\ddot{\phi} s^{2}+2 \ddot{\phi}\left(-s^{\prime \prime}+\mu q s^{\prime}\right) .
$$

Then, from (2.11) and the fact that $\phi^{\prime} \leq 0$, we have

$$
\ddot{\phi}\left(s_{0}\right)=-\frac{1}{s^{\prime 2}} \lambda_{1}^{c} \phi^{\prime}\left(s_{0}\right)=-\frac{1}{s^{\prime}} \lambda_{1}^{c} \phi^{\prime}\left(s_{0}\right)>0,
$$


which implies that $\dot{\phi}$ has a minimum at $s_{0}$, in contradiction with the foregoing assertion. Then $\ddot{\phi}(s) \geq 0$ for every $s$. This completes the proof of (3.1).

If $\lambda_{1}(\Omega)=\lambda_{1}\left(B_{a}^{\lambda, q}\right)$, then $\phi$ must be a $\lambda_{1}(\Omega)$-eigenfunction ([3, page 1057]) and (3.2) must be an equality, which implies that $\overline{\operatorname{grad}} r$ is tangent to $P$ (that is, all the geodesic segments of $M$ starting from $P$ and reaching a point $m$ in $\Omega$ are in $P$ ) and $S(r)$, restricted to the intersection of the geodesic sphere $\partial B_{r}(p)$ of centre $p$ and radius $r$ in $M$ and $P$, is equal to $S_{\lambda, q}(r)$, the Weingarten map of a geodesic sphere in $\mathbb{K}^{q}(\lambda)$. Moreover, if $S^{P}(r)$ is the Weingarten map of $\partial B_{r}(p) \cap P$ in $P$ and $N$ is the unit normal vector to $\partial B_{r}(p) \cap P$ in $P$, we have

$$
\left\langle S^{P}(r) X, Y\right\rangle=\left\langle\nabla_{X} Y, N\right\rangle=\left\langle\bar{\nabla}_{X} Y-\alpha(X, Y), N\right\rangle=\langle S(r) X, Y\rangle,
$$

because $\alpha(X, Y)$ is orthogonal to $P$ and $N$ is tangent to $P$. Then, $S^{P}(r)=$ $S_{\lambda, q}(r)$, which implies ([11], [4]) that $\Omega=B_{a}(p)$ is holomorphically isometric to $B_{a}^{\lambda, q}$.

Now, from Gauss equation, if $R^{P}$ is the curvature tensor of $P$,

$$
\begin{aligned}
R_{\gamma^{\prime} X \gamma^{\prime} X} & =R_{\gamma^{\prime} X \gamma^{\prime} X}^{P}+\left\langle\alpha\left(X, \gamma^{\prime}\right), \alpha\left(X, \gamma^{\prime}\right)\right\rangle-\left\langle\alpha\left(\gamma^{\prime}, \gamma^{\prime}\right), \alpha(X, X)\right\rangle \\
& =R_{\gamma^{\prime} X \gamma^{\prime} X}^{P}+\left\langle\alpha\left(X, \gamma^{\prime}\right), \alpha\left(X, \gamma^{\prime}\right)\right\rangle .
\end{aligned}
$$

But $R(t) \leq R_{\lambda}(t)$ and, from the equality, $R(t)=R_{\lambda}(t)=R^{P}(t)$. Then, $\alpha\left(X, \gamma^{\prime}\right)=0$ for every $X$, that is, $\left\langle L_{\xi} X, \gamma^{\prime}\right\rangle=0$, for every $\xi$ orthogonal to $P$, and every $X$ tangent to $P$. If the hypothesis $R(t) \leq R_{\lambda}(t)$ holds for every $p \in \Omega$, then, from (3.15) and the fact that $R^{P}(t)=R_{\lambda}(t)$, we get $R(t)=R_{\lambda}(t)$ and $\alpha\left(X, \gamma^{\prime}\right)=0$ for every $p$ in $\Omega$, and then $\alpha=0$ on $\Omega$.

\section{Bounds for the sectional curvatures of $\mathbb{K}^{m}(v) \times \mathbb{K}^{r}(v)$}

Within this paragraph, $\lambda>0$. Let us consider $M=\mathbb{K}^{m}(\nu) \times \mathbb{K}^{r}(\nu)$, $p=(a, b) \in \mathbb{K}^{m}(v) \times \mathbb{K}^{r}(v)$, and a geodesic $\gamma(t)$, starting from $p$, with $\gamma^{\prime}(t)=A(t)+B(t)$, and $J \gamma^{\prime}(t)=J_{1} A(t)+J_{2} B(t)$, where $A(t)$ (respectively $B(t)$ ) is tangent to $\mathbb{K}^{m}(v)$ (respectively $\mathbb{K}^{r}(\nu)$ ), $J_{1}$ (respectively $J_{2}$ ) being the complex structure on $\mathbb{K}^{m}(\nu)$ (respectively $\mathbb{K}^{r}(\nu)$ ). Let $X$ be a unit vector field along $\gamma$, orthogonal to $\gamma^{\prime}$, and let us write $X=X_{1}+X_{2}$, where $X_{1}$ (respectively $\left.X_{2}\right)$ is tangent to $\mathbb{K}^{m}(v)$ (respectively $\mathbb{K}^{r}(v)$ ). Then $X_{1}=\mu_{1} J_{1} A+Y_{1}$ and $X_{2}=\mu_{2} J_{2} B+Y_{2}$, with $Y_{1}$ (respectively $Y_{2}$ ) orthogonal to $J_{1} A$ (respectively $\left.J_{2} A\right)$, and

$$
\begin{aligned}
\langle R(t) X, X\rangle & =\left\langle R(t)\left(X_{1}+X_{2}\right), X_{1}+X_{2}\right\rangle=R_{A X_{1} A X_{1}}+R_{B X_{2} B X_{2}} \\
& =R_{A \mu_{1} J_{1} A A \mu_{1} J_{1} A}+R_{A Y_{1} A Y_{1}}+R_{B \mu_{2} J_{2} B B \mu_{2} J_{2} B}+R_{B Y_{2} B Y_{2}}
\end{aligned}
$$




$$
\begin{aligned}
& =4 v\left(\mu_{1}^{2}|A|^{4}+\mu_{2}^{2}|B|^{4}\right)+v\left(|A|^{2}\left|Y_{1}\right|^{2}+|B|^{2}\left|Y_{2}\right|^{2}-\left\langle A, Y_{1}\right\rangle^{2}-\left\langle B, Y_{2}\right\rangle^{2}\right) \\
& \leq 4 v\left(\mu_{1}^{2}|A|^{4}+\mu_{2}^{2}|B|^{4}\right)+v\left(\left|Y_{1}\right|^{2}+\left|Y_{2}\right|^{2}\right) \equiv f,
\end{aligned}
$$

since $\left|\gamma^{\prime}\right|=1$ and $|X|=1$ imply that $|A|,|B|,\left|Y_{1}\right|$ and $\left|Y_{2}\right|$ are less than or equal to 1 . Similarly,

$$
\begin{aligned}
\left\langle R_{\lambda}(t) X, X\right\rangle & =\lambda+3 \lambda\left\langle X, J \gamma^{\prime}\right\rangle^{2} \\
& =\lambda+3 \lambda\left(\mu_{1}|A|^{2}+\mu_{2}|B|^{2}\right)^{2}+\lambda\left(\left|Y_{1}\right|^{2}+\left|Y_{2}\right|^{2}\right) \equiv g .
\end{aligned}
$$

Then we have to study the values of $v \leq \lambda$ for which $g-f \geq 0$, but

$$
g-f \geq h=\lambda+(3 \lambda-4 v)\left(\mu_{1}^{2}|A|^{4}+\mu_{2}^{2}|B|^{4}\right)+6 \lambda \mu_{1}|A|^{2} \mu_{2}|B|^{2},
$$

then it will be enough to prove that $h \geq 0$ for those values of $\nu$.

Since $\gamma^{\prime}$ and $X$ are unit vectors, we have

$$
\mu_{1}^{2}|A|^{2}+\mu_{2}^{2}|B|^{2} \leq 1 \quad \text { and } \quad|A|^{2}+|B|^{2}=1 .
$$

If we put $|A|=\cos u,|B|=\sin u, \mu_{1}|A|=\alpha, \mu_{2}|B|=\beta, h$ will be nonnegative if the infimum of the function

$$
h(\alpha, \beta, u)=\lambda+(3 \lambda-4 \nu)\left(\alpha^{2} \cos ^{2} u+\beta^{2} \sin ^{2} u\right)+6 \lambda \alpha \cos u \beta \sin u
$$

on the domain

$$
D=\left\{(\alpha, \beta, u) / \alpha^{2}+\beta^{2} \leq 1 \text { and } u \in[0,2 \pi]\right\}
$$

is nonnegative.

Let $w$ and $\phi$ be the functions defined by $w(x, y)=\lambda+(3 \lambda-4 v)\left(x^{2}+y^{2}\right)+6 \lambda x y$ and $\phi(\alpha, \beta, u)=(\alpha \cos u, \beta \sin u)$, then

$$
\inf _{D} h=\inf _{\phi(D)} w
$$

and

$$
\begin{aligned}
\phi(D) & =\{(r \cos u \cos v, r \sin u \sin v) / r \in[0,1], u, v \in[0,2 \pi]\} \\
& =\left\{(x, y) \in \mathbb{R}^{2} /|x|+|y| \leq 1\right\} .
\end{aligned}
$$

If $2 \nu \neq 3 \lambda$, the function $w$ has only a critical point in $\phi(D)$, which is a maximum. Then the minimum of $w$ is attained on the boundary of $\phi(D)$. If we evaluate $w$ on this boundary, we see that the minimum of $w$ is attained at the points $(-1 / 2,1 / 2)$ and $(1 / 2,-1 / 2)$, and its value is $\lambda-2 v$, then it is nonnegative for $v \leq \lambda / 2$. Then 
PROPOSITION 4,1. The manifolds $\mathbb{K}^{m}(v) \times \mathbb{K}^{r}(v)$, with $m+r=n$ and $\lambda / 4<$ $v \leq \lambda / 2$ satisfy the condition $R(t) \leq R_{\lambda}(t)$ and the maximum value of their sectional curvatures is $4 v>\lambda$.

\section{References}

[1] S. J. Bang, 'The first Dirichlet eigenvalue and radius of a geodesic ball', Proc. Amer. Math. Soc. 104 (1988), 885-886.

[2] I. Chavel, Eigenvalues in Riemannian geometry (Academic Press, New York, 1984).

[3] S. Y. Cheng, P. Li and S. T. Yau, 'Heat equations on minimal submanifolds and their applications', Amer. J. Math. 106 (1984), 1033-1065.

[4] F. Giménez, 'Comparison theorems for the volume of a complex submanifold of a Kaehler manifold', Israel J. Math. 71 (1990), 239-255.

[5] F. Giménez and V. Miquel, 'Bounds for the first Dirichlet eigenvalue of domains in kaehler manifolds', Archiv der Mathematik 56 (1991), 370-375.

[6] A. Gray, Tubes (Addison-Wesley, New York, Reading, 1990).

[7] R. E. Greene and H. Wu, Function theory on manifolds which possess a pole, Lecture in Mathematics 699 (Springer-Verlag, 1979).

[8] L. Jorge and D. Koutroufiotis, 'An estimate for the curvature of bounded submanifolds', Amer. J. Math. 103 (1981), 711-725.

[9] H. Karcher, 'Riemannian comparison constructions', in: Global differential geometry (ed. S. S. Chern) (Math. Assoc. Amer., Washington, D.C., 1989) pp. 170-222.

[10] S. Kobayashi and K. Nomizu, Foundations of Differential Geometry, volume II (Interscience, New York, 1969).

[11] O. Kowalski and L. Vanhecke, 'A new formula for the shape operator of a geodesic sphere and its applications', Math. Zeit. 192 (1986), 613-625.

[12] S. M. Markvorsen, 'On the heat kernel comparison theorems for minimal submanifolds', Proc. Amer. Math. Soc. 97 (1986), 479-482.

[13] H. L. Royden, 'Comparison theorems for the matrix Riccati equation', Comm. Pure Appl. Math. 41 (1988), 739-746.

Dept. de Geometría y Topología

Universidad de Valencia

46100 Burjasot (Valencia)

Spain
Dept. de Mathemática Aplicada E.T.S.I Industriales Universidad Politécnica de Valencia Valencia Spain

\footnotetext{
Dept. de Geometría y Topología

Universidad de Valencia

46100 Burjasot (Valencia)

Spain
} 